\title{
PAS-positive lymphocyte vacuoles can be used as diagnostic screening test for Pompe disease
}

\author{
Marloes L. C. Hagemans • Rolinda L. Stigter • Carine I. van Capelle • \\ Nadine A. M. E. van der Beek • Leon P. F. Winkel • Laura van Vliet • Wim C. J. Hop • \\ Arnold J. J. Reuser • Auke Beishuizen • Ans T. van der Ploeg
}

Received: 6 September 2009 /Revised: 4 December 2009 / Accepted: 7 December 2009/Published online: 27 January 2010

(C) The Author(s) 2010. This article is published with open access at Springerlink.com

\begin{abstract}
Screening of blood films for the presence of periodic acid-Schiff (PAS)-positive lymphocyte vacuoles is sometimes used to support the diagnosis of Pompe disease, but the actual diagnostic value is still unknown. We collected peripheral blood films from 65 untreated Pompe patients and
\end{abstract}

Communicated by: Ed Wraith

Competing interest: None declared.

M. L. C. Hagemans · C. I. van Capelle · L. P. F. Winkel •

A. T. van der Ploeg

Department of Paediatrics, Division of Metabolic Diseases and

Genetics, Erasmus MC University Medical Center- Sophia

Children's Hospital,

Rotterdam, The Netherlands

R. L. Stigter · A. Beishuizen

Department of Paediatric Oncology/Hematology, Erasmus MC

University Medical Center- Sophia Children's Hospital,

Rotterdam, The Netherlands

N. A. M. E. van der Beek

Department of Neurology,

Erasmus MC University Medical Center,

Rotterdam, The Netherlands

L. van Vliet • A. J. J. Reuser

Department of Clinical Genetics,

Erasmus MC University Medical Center,

Rotterdam, The Netherlands

W. C. J. Hop

Department of Biostatistics,

Erasmus MC University Medical Center,

Rotterdam, The Netherlands

M. L. C. Hagemans $(\square)$

Department of Paediatrics,

Division of Metabolic Diseases and Genetics,

Erasmus MC-Sophia,

Dr. Molewaterplein 60,

3015 GJ Rotterdam, The Netherlands

e-mail:m.hagemans@erasmusmc.nl
51 controls. Lymphocyte vacuolization was quantified using three methods: percentage vacuolated lymphocytes, percentage PAS-positive lymphocytes, and a PAS score depending on staining intensity. Diagnostic accuracy of the tests was assessed using receiver operating characteristic (ROC) curves. All three methods fully discerned classic infantile patients from controls. The mean values of patients with milder forms of Pompe disease were significantly higher than those of controls, but full separation was not obtained. The area under the ROC curve was 0.98 for the percentage vacuolated lymphocytes (optimal cutoff value 3 ; sensitivity $91 \%$, specificity $96 \%$ ) and 0.99 for the percentage PASpositive lymphocytes and PAS score (optimal cutoff value 9; sensitivity $100 \%$, specificity $98 \%$ ). Our data indicate that PAS-stained blood films can be used as a reliable screening tool to support a diagnosis of Pompe disease. The percentage of PAS-positive lymphocytes is convenient for use in clinical practice but should always be interpreted in combination with other clinical and laboratory parameters.

$\begin{array}{ll}\text { Abbreviations } \\ \text { ALAT } & \text { Alanine aminotransferase } \\ \text { ASAT } & \text { Aspartate aminotransferase } \\ \text { CK } & \text { Creatine kinase } \\ \text { LAMP2 } & \text { Lysosomal-associated membrane protein } 2 \\ \text { MGG } & \text { May-Grünwald/Giemsa } \\ \text { PAS } & \text { Periodic acid-Schiff } \\ \text { ROC } & \text { Receiver-operating characteristic }\end{array}$

\section{Introduction}

Pompe disease (glycogen storage disease type II, acid maltase deficiency; OMIM \# 232300) is an inherited 
metabolic disorder in which a deficiency of the lysosomal enzyme acid $\alpha$-glucosidase (EC 3.2.1.3/20) causes glycogen storage. This leads to a continuum of phenotypes with the rapidly progressive classic infantile form, which is characterized by cardiomyopathy and generalized hypotonia at the most severe end. Milder forms of the disease may present at any age, with limb-girdle weakness as the most prominent clinical feature (Hirschhorn and Reuser 2000; van der Ploeg and Reuser 2008). The diagnosis of Pompe disease is confirmed by measuring acid $\alpha$-glucosidase activity in leukocytes using the latest optimized procedures, or in cultured fibroblasts (Hirschhorn and Reuser 2000; van Diggelen et al. 2009; Winchester et al. 2008). These tests, however, are carried out in a specialized laboratory and are usually not applied unless there is already a strong suspicion for Pompe disease. A reliable and simple test to include or exclude Pompe disease in the differential diagnosis would be of help to the clinician.

Glycogen storage in Pompe disease is found in lysosomes throughout the body, including lymphocytes in peripheral blood (de Barsy and Hers 1975; Ikeda et al. 1982; Trend et al. 1985). Peripheral blood is easy to obtain, and the presence of glycogen-filled vacuoles in lymphocytes can be detected by microscopic inspection of blood films. As early as 1977, the potential usefulness of this test on the path to diagnosing Pompe disease was discussed (von Bassewitz et al. 1977). In current clinical practice, it is sometimes used as a quick screening method before final confirmation by enzymatic or genetic analysis (Muntoni et al. 2005; Straub 2008a) or as a secondary test to support a diagnosis of Pompe disease (Winchester et al. 2008), but the test was never formally validated.

Vacuolated lymphocytes - in essence, lysosomes filled with nondegraded material - occur in many storage disorders. In a retrospective review of 2,550 blood films of patients with a history suggestive of metabolic disease (Anderson et al. 2005), vacuolated lymphocytes were identified in 156 cases. Twenty-three percent of those were from patients with Pompe disease: $15 \%$ for the rapidly progressive classic infantile form and $8 \%$ for milder forms presenting in childhood, adolescence, or adulthood. Other diagnoses included juvenile Batten's disease (31\%), GM1 gangliosidosis $(9 \%)$, galactosialidosis $(5 \%)$, mucopolysaccharidosis $(5 \%)$, and I-cell disease (4\%). Pompe disease was the only disease in which lymphocytes were reported to stain positively with periodic acid-Schiff (PAS) reagent (Anderson et al. 2005). Based on the literature, the presence of PAS-positive lymphocyte vacuoles seems to be specific for Pompe disease (Anderson et al. 2005; de Barsy \& Hers 1975; Ikeda et al. 1982; Straub 2008a; Trend et al. 1985), but it was also noted that vacuolated lymphocytes are less frequent in milder forms of the disease compared with the classic infantile form (Anderson et al. 2005). In fact, the actual diagnostic value of the test remains unknown unless the frequency in Pompe disease is compared with a control group.

We have collected peripheral blood films since 1999 as part of the clinical and laboratory evaluation of patients with Pompe disease. To investigate the diagnostic value of the test for both classic infantile and milder forms, we quantified the percentage of vacuolated lymphocytes, the percentage of PAS-positive lymphocytes, and the intensity of PAS staining in a group of 65 patients and 51 controls.

\section{Methods}

Patients

Between January 1999 and December 2006, blood films were obtained from 65 untreated patients: 8 babies with classic infantile Pompe disease and 16 children and 41 adults with milder forms. Patients participated in studies investigating the natural course of Pompe disease or the effects of enzyme replacement therapy. All studies were approved by the Medical Ethical Committee of Erasmus MC, the Netherlands, and written informed consent was obtained from patients and/or parents. In all patients, the diagnosis of Pompe disease was confirmed by deficiency of acid $\alpha$-glucosidase activity in cultured fibroblasts or leukocytes (Hirschhorn and Reuser 2000; Okumiya et al. 2006). From all patients, one or more blood films were obtained before the start of enzyme replacement therapy and from 16 patients during treatment.

\section{Controls}

Control values were obtained from 17 children visiting the outpatient clinic of the departments of Metabolic Diseases and Genetics, Endocrinology, or Gastro-enterology (Erasmus MC-Sophia Children's Hospital) and from whom a blood sample was needed for routine clinical care. Exclusion criteria were suspected or confirmed diagnosis of a lysosomal or glycogen storage disorder, acute infection, or other diseases that might affect the lymphocytes. Adult control values were obtained from 34 healthy blood donors (Sanquin Blood Bank, Rotterdam, the Netherlands). The Medical Ethical Committee of Erasmus MC approved the protocol to obtain control samples. Informed consent was obtained from children and their parents, as well as from all blood donors.

\section{Lymphocyte scores}

One blood sample was taken from every patient, and six blood films were prepared. Two were stained with May- 
Grünwald/ Giemsa (MGG) reagent, two with PAS reagent, and two were extras. All blood films were made within $2 \mathrm{~h}$ of the sample being drawn. Staining and microscopic inspection of the blood films was done within 1-5 days. To quantify the degree of lymphocyte vacuolization, three methods were used. First, the number of vacuolated lymphocytes per 100 lymphocytes (percentage vacuolated lymphocytes) was counted in the MGG-stained blood films. For the second and third methods, PAS reagent was used to stain for the presence of glycogen. The number of PASpositive lymphocytes per 100 lymphocytes was then counted (percentage PAS-positive lymphocytes). Finally, the presence of glycogen-filled vacuoles was quantified by a PAS intensity score. This scoring method was originally developed to quantify lymphocytic proliferation in leukemia (Bain 1995; Hayhoe and Quaglino 1980; Mitus et al. 1958). Each lymphocyte received a score varying from 1 (a small PAS-positive spot) to 4 (a large group of large PASpositive spots). Lymphocytes without PAS-positive vacuoles were scored 0 . The sum of the scores of 100 lymphocytes (PAS score) was then calculated. All scoring was performed in duplicate (two different blood films by two different observers), and the average of the two scores was used in the analyses. Figure 1 shows photomicrographs of blood films from patients with Pompe disease and a healthy control.
Statistical analysis

Patients were subdivided in three groups: patients with the severe classic infantile form of Pompe disease, children under 18 years of age with delayed onset of symptoms, and patients of 18 years and older. Likewise, controls were divided in three subgroups: up to age 1 , between 1 and 18 years, and 18 years and older. Age, sex, percentage of vacuolated lymphocytes, percentage PAS-positive lymphocytes, and PAS score of patients and controls were summarized using median, ranges, and percentages. Differences between patient and control groups were compared with Pearson's $\chi^{2}$ test (ratio men/women) and the Mann-Whitney test (age). Differences between groups in percentage of vacuolated lymphocytes, percentage PAS-positive lymphocytes, and PAS score were tested with the Mann-Whitney test. When more than one blood film per patient was available, the most recent measurement before starting enzyme therapy was used in the analyses.

To quantify the diagnostic accuracy of the test, receiveroperating characteristic (ROC) curves were made for the percentage of vacuolated lymphocytes, percentage PASpositive lymphocytes, and total PAS score. ROC curves plot the true-positive rate (sensitivity) against the falsepositive rate (1- specificity) for all possible cutoff values.
Fig. 1 Photomicrographs of blood films from patients with Pompe disease and a healthy control. a May-Grünwald/ Giemsa-stained blood film of a late-onset Pompe patient showing a lymphocyte with two distinct vacuoles; $\mathbf{b}$ periodic acid-Schiff (PAS)-stained blood film of a healthy control (PAS score $=0$ ); c PAS-stained blood film of a late-onset Pompe patient showing a lymphocyte with PAS-positive inclusions (PAS score $=2$ ); d PAS-stained blood film of a patient with classic infantile Pompe disease showing a lymphocyte with a larger number of PAS-positive inclusions (PAS score $=3$ )
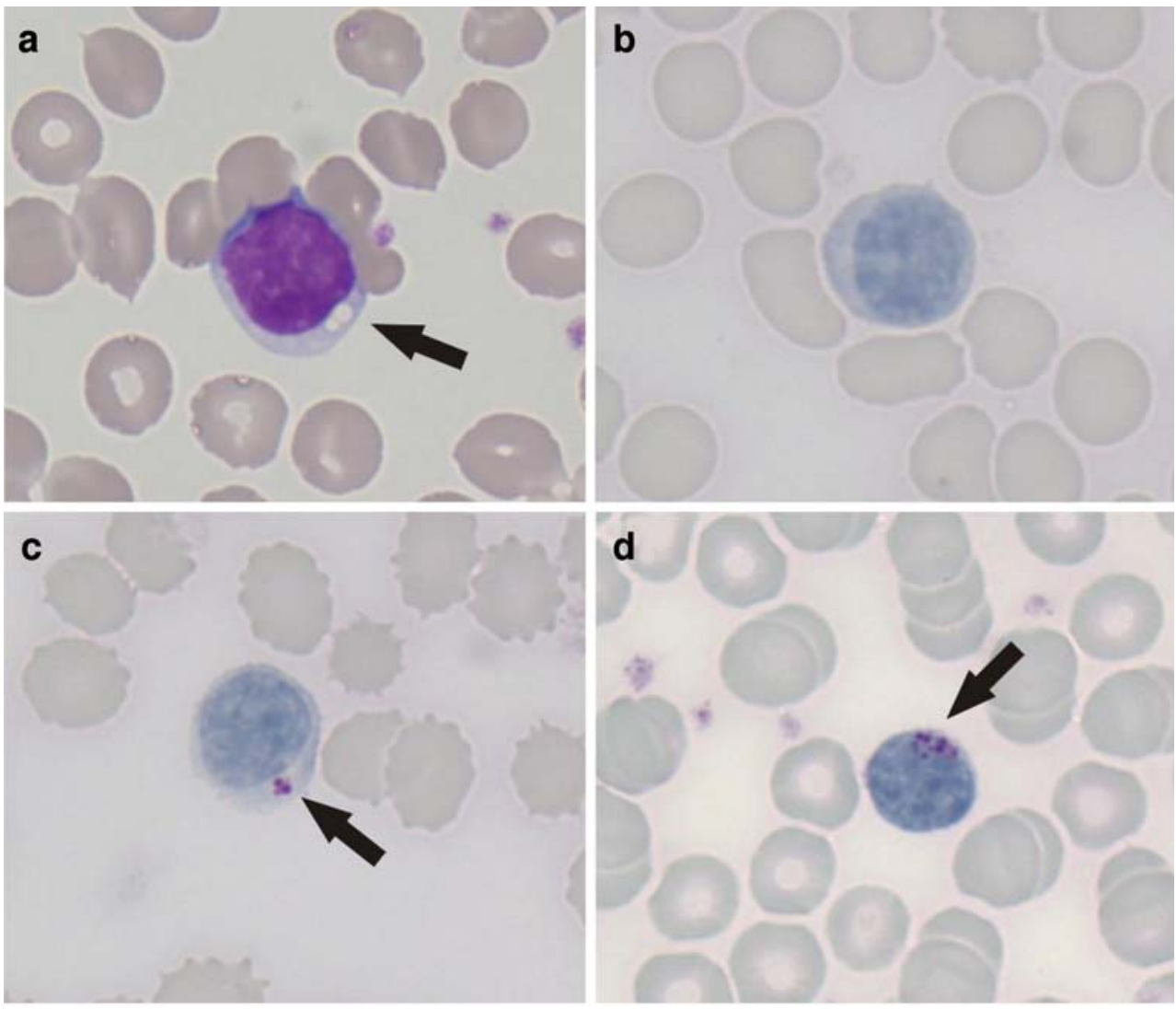
The area under each ROC curve was calculated and compared between the three tests.

The intraclass correlation coefficient was used to assess agreement between repeated measurements within patients from whom two or more samples were available before start of enzyme therapy. The relation between test scores and age was evaluated with Spearman correlation coefficients. Differences in test scores between male and female patients were tested with Mann-Whitney tests. All analyses were performed using SPSS for Windows (version 15.0, SPSS Inc. Chicago, IL, USA). A $p$ value $<0.05$ was considered significant.

\section{Results}

\section{General characteristics}

Table 1 shows the general characteristics of the patient and control groups. The ratio between the number of infants, children, and adults was 1:2:5 in the patient group and 1:3:8 in the control group. When the groups were compared, no significant differences were found in age and male/female ratio.

\section{Test scores}

Table 2 shows the percentage of vacuolated lymphocytes, percentage of PAS-positive lymphocytes, and PAS scores per subgroup of patients and controls. In Fig. 2, these scores are plotted for classic infantile patients, children and adults with Pompe disease, and controls. The figure shows that all three methods fully discriminate between classic infantile patients and controls. Values of older children and adults with Pompe disease differed significantly from those of controls $(p<0.001$ for all three methods), but full separation was not obtained. Classic infantile patients scored significantly higher (all $p<0.001$ ) than children and adults with milder forms of the disease.

\section{Diagnostic accuracy}

Diagnostic accuracy of the three tests was assessed using ROC curves. With all patients and controls taken together, the area under the ROC curve was 0.98 for the percentage of vacuolated lymphocytes, and 0.99 for the percentage of PAS-positive lymphocytes and PAS-score (Fig. 3). Because classic infantile patients showed no overlap with controls, we also calculated ROC curves only for children and adults with milder forms of the disease and controls $>1$ year. Results were comparable: the area under the curve was 0.97 for the percentage of vacuolated lymphocytes and 0.99 for the percentage of PAS-positive lymphocytes and the PAS score. ROC curves were then used to determine the optimal cutoff value to discern between patients and controls. Again, results for the total group were comparable with those excluding classic infantile patients and controls $<1$ year. Optimal cutoff values (upper left corner of the curves) were determined as 3 for the percentage of vacuolated lymphocytes (i.e., a score $\geq 3$ indicates a patient; sensitivity $91 \%$, specificity $96 \%$ ) and 9 for the percentage

Table 1 General characteristics of patients with Pompe disease and controls

\begin{tabular}{lccc}
\hline & Number of patients & Age [median (range) & Male/female (\%) \\
\hline $\begin{array}{l}\text { Pompe patients } \\
\text { Classic infantile }\end{array}$ & 8 & & $4(0-10)$ months \\
Milder form, $<18$ years & 16 & $12(3-17)$ years & $50 / 50$ \\
Milder form, $\geq 18$ years & 41 & $49(24-72)$ years & $69 / 31$ \\
Total & 65 & $38(0-72)$ years & $51 / 49$ \\
Controls & 4 & $9(0-12)$ months & $55 / 45$ \\
$\leq 1$ year & & $10(3-15)$ years & $75 / 25$ \\
$1-18$ years & 13 & $58(20-68)$ years & $62 / 38$ \\
$\geq 18$ years & 34 & $43(0-68)$ years & $53 / 47$ \\
Total & 51 & $57 / 43$ \\
\hline
\end{tabular}

${ }^{a}$ Reasons for visiting the outpatient clinic of the Department of Metabolic Diseases and Genetics: biotinidase deficiency $(n=2)$, phenylketonuria $(n=1)$, suspected galactosemia $(n=1)$

${ }^{\mathrm{b}}$ Reasons for visiting the outpatient clinic of the Department of Metabolic Diseases and Genetics: hypercholesterolemia ( $\left.n=1\right)$, lipoprotein lipase deficiency $(n=1)$, ornithine transcarbamylase deficiency $(n=2)$, phenylketonuria $(n=2)$, tyrosinemia $(n=2)$; Department of Endocrinology: adrenogenital syndrome $(n=2)$, growth hormone deficiency $(n=1)$, growth retardation $(n=1)$; Gastroenterology: unknown reason $(n=1)$.

${ }^{\mathrm{c}}$ Healthy controls donating blood on a regular basis 
Table 2 Vacuolated lymphocytes, periodic acid-Schiff (PAS)-positive lymphocytes, and PAS scores of patients with Pompe disease and controls

\begin{tabular}{lclll}
\hline & Number & $\begin{array}{l}\text { Percent vacuolated lymphocytes } \\
{[\text { median (range) }}\end{array}$ & $\begin{array}{l}\text { Percent PAS-positive lymphocytes } \\
{[\text { median (range) }}\end{array}$ & $\begin{array}{l}\text { PAS score } \\
{[\text { median (range) }]}\end{array}$ \\
\hline $\begin{array}{l}\text { Pompe patients } \\
\text { Classic infantile }\end{array}$ & 8 & $56(25-79)$ & $82(40-96)$ & $123(77-163)$ \\
Milder form, $<18$ years & 16 & $10(0-40)$ & $37(10-67)$ & $40(10-87)$ \\
Milder form, $\geq 18$ years & 41 & $9(1-52)$ & $39(16-58)$ & $40(16-68)$ \\
Total & 65 & $10(0-79)$ & $2(1-96)$ & $45(10-163)$ \\
Controls & & & $2(0-7)$ & $2(1-3)$ \\
$\leq 1$ year & 4 & $0(0-0)$ & $3(0-15)$ & $3(0-8)$ \\
$1-18$ years & 13 & $0(0-1)$ & $2(0-15)$ & $2(0-19)$ \\
$\geq 18$ years & 34 & $1(0-3)$ & $0(0-3)$ & \\
Total & 51 & & & \\
\hline
\end{tabular}

of PAS-positive vacuoles and PAS score (sensitivity $100 \%$, specificity $98 \%$ ).

\section{Within-patient variability}

The study population included 26 patients with two or more subsequent measurements before the start of enzyme replacement therapy. Measurements, with intervals varying between 5 days and 17.7 months (median 5.8 months), showed moderate agreement: the intraclass correlation coefficient was 0.69 for the percentage of vacuolated lymphocytes, 0.62 for the percentage of PAS-positive lymphocytes, and 0.68 for PAS-score.

\section{Relationship with age and sex}

No correlations between the percentage of vacuolated lymphocytes, percentage of PAS-positive lymphocytes, PAS-score, and age were found in subgroups with milder forms of Pompe disease. For classic infantile patients, the percentage of PAS-positive lymphocytes increased with age $\left(r_{\mathrm{s}}=0.71, p=0.049\right)$. A small but statistically significant correlation with age was also present in the control group $\left(r_{\mathrm{s}}=0.33,0.35\right.$ and 0.34 ; all $\left.p<0.02\right)$. No significant differences in test scores between male and female patients were found in any of the subgroups or the control group.

\section{Effect of enzyme replacement therapy on PAS score}

For 16 of the 65 patients, blood films from shortly (maximum 3 weeks) before and after the start of treatment were available. In all patients, the percentage of vacuolated lymphocytes, percentage of PAS-positive lymphocytes, and PAS score decreased greatly within a few weeks after the start of enzyme replacement therapy to values in or approximating the control range.

\section{Discussion}

We investigated whether quantification of glycogen-filled vacuoles in peripheral blood lymphocytes would be of use as a simple screening method to include or exclude Pompe disease in the differential diagnosis. To this end, peripheral blood films were collected from 65 patients with Pompe disease and 51 controls. Using routine staining procedures for light microscopy, the percentage of vacuolated lymphocytes was calculated. By staining the glycogen with PAS reagent, two additional values were obtained: the percentage of PAS-positive lymphocytes and a PAS score quantifying staining intensity. All three methods were able to discern classic infantile Pompe patients from controls without Pompe disease. Median scores of children and adults with milder forms of the disease were also clearly higher than those of the control group, but some overlap still existed. Nevertheless, diagnostic accuracy of the tests was very good, with areas under the ROC curve approaching $100 \%$ for all three methods. Although all three methods showed excellent results, the percentage of PAS-positive lymphocytes and PAS score performed slightly better than the percentage of vacuolated lymphocytes. Moreover, the PAS reagent used for the first two measures specifically stains glycogen accumulation, which is a hallmark of Pompe disease. As the diagnostic accuracy was comparably high for both the percentage of PAS-positive lymphocytes and PAS score, it can be concluded that stain-intensity quantification by adding a score to the PAS-stained lymphocytes is not necessary.

With a cutoff value of 9 for the percentage of PASpositive lymphocytes, a sensitivity of $100 \%$ and a specificity of $98 \%$ were reached. The $100 \%$ sensitivity means that no patients should be missed; a specificity of $98 \%$ means that the test is likely to give a false positive result in $2 \%$ of cases. The latter will be detected when further diagnostic 


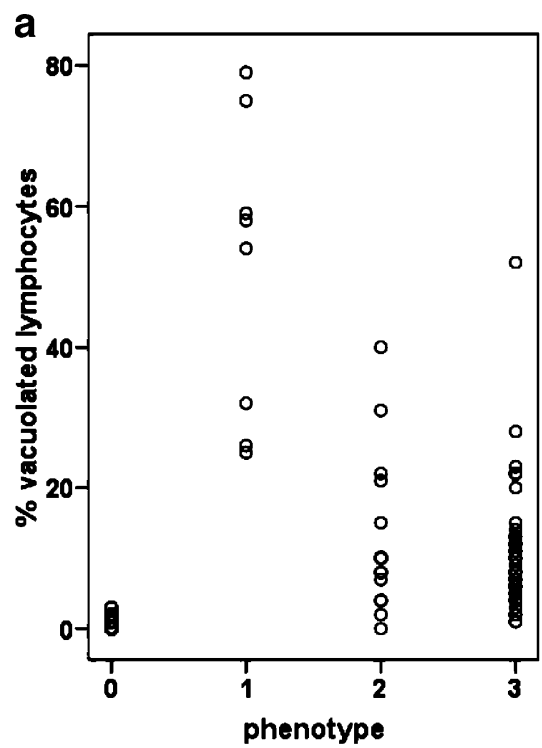

b
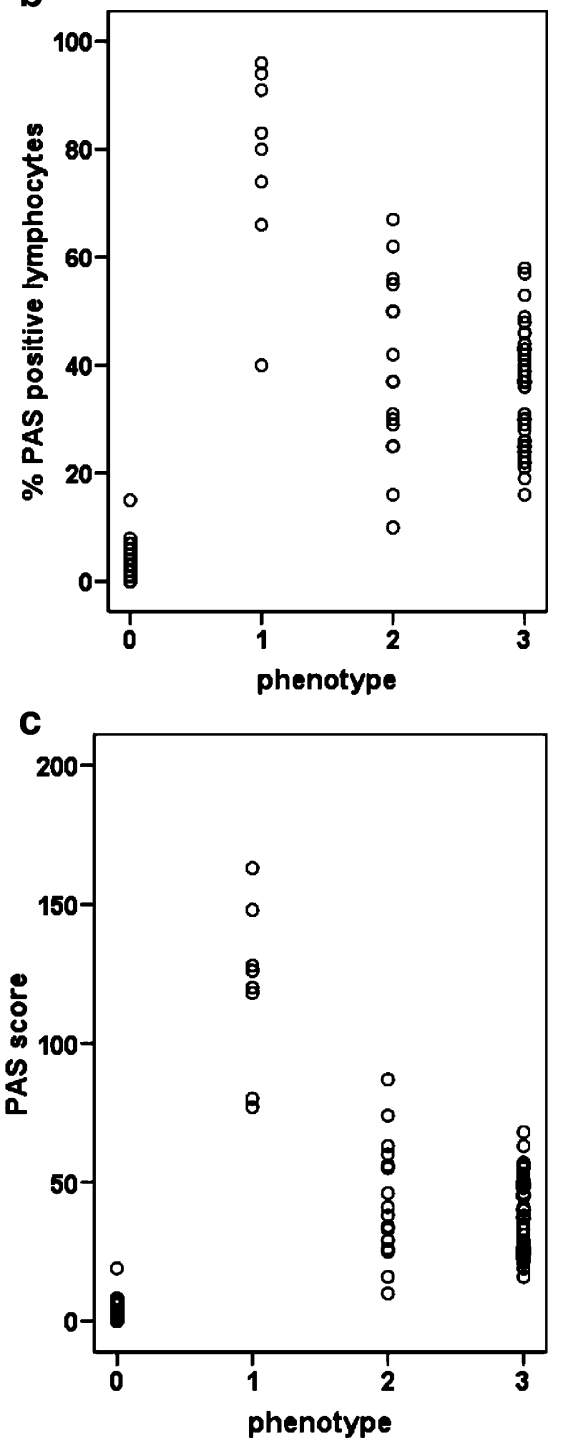

Fig. 2 Dot plots of a percentage of vacuolated lymphocytes, b percentage periodic acid-Schiff (PAS)-positive lymphocytes, and c PAS scores of 65 patients with Pompe disease and 51 controls. 0 controls $(n=51) ; 1$ classic infantile patients $(n=8) ; 2$ children with Pompe disease $(n=16) ; 3$ adults with Pompe disease $(n=41)$

testing is performed and the acid $\alpha$-glucosidase activity turns out to be within the normal range. It should be noted, however, that we found considerable within-patient variability in subsequent measurements. Some variability between observers and laboratories is likely to exist as well. In clinical practice, a low percentage of PAS-positive lymphocyte vacuoles should therefore not automatically exclude the possibility of Pompe disease, especially when the patient is an adolescent or adult. The test result should always be considered in combination with other resultsfor example, creatine kinase (CK), alanine aminotransferase (ALAT), and aspartate aminotransferase (ASAT) (Winkel et al. 2005) - and a careful clinical examination. On the other hand, when a high percentage of PAS-positive lymphocyte vacuoles is found, further testing by measuring acid $\alpha$ glucosidase activity in leukocytes (van Diggelen et al. 2009) is always indicated. This fits into previously proposed diagnostic algorithms for childhood- and adultonset Pompe disease (Straub 2008b).

Another issue that should be addressed is our choice of the control group - which consisted of children without Pompe disease, lysosomal disease, or glycogen storage

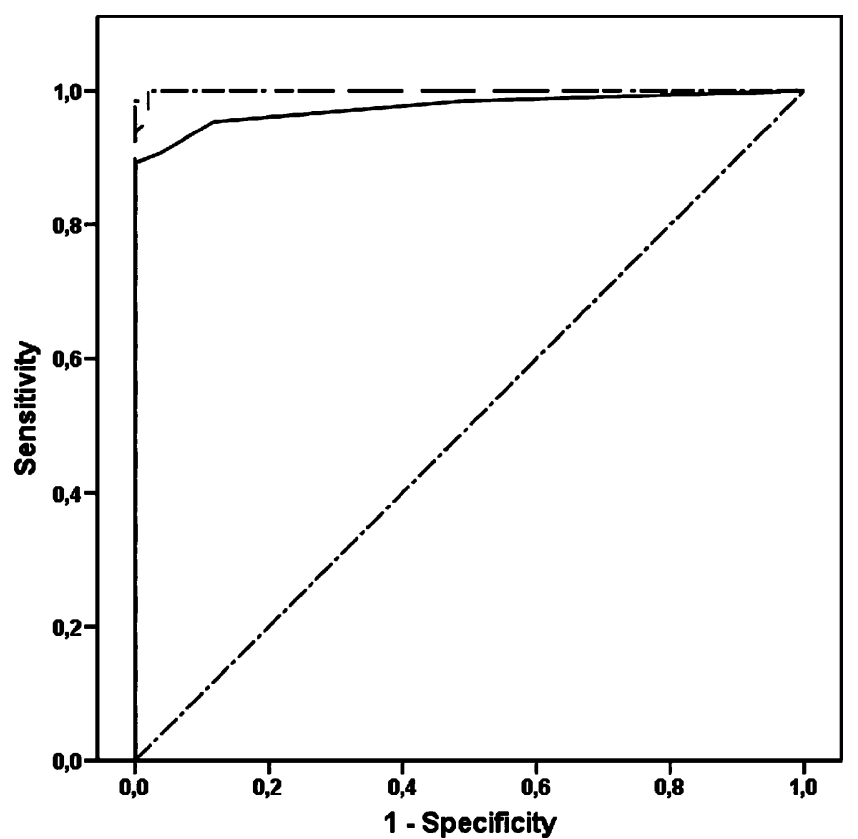

Fig. 3 Receiver operating characteristic (ROC) curves for the percentage of vacuolated lymphocytes, percentage of periodic acidSchiff (PAS)-positive lymphocytes, and PAS-score of 65 patients with Pompe disease and 51 controls. - percentage of vacuolated lymphocytes; $\bullet \ldots \ldots$ - $\ldots$ percentage of PAS positive lymphocytes;

PAS score; $\cdots$... reference line 
disease and healthy adult controls. It could be argued that in most cases of suspected Pompe disease, one would rather want to discern patients with Pompe disease from patients with other diseases causing generalized weakness and hypertrophic cardiomyopathy (infants) or limb-girdle weakness (children, adolescents, and adults). To our knowledge PAS-positive vacuolar pathology in peripheral lymphocytes is not described for such disorders, although it could perhaps be expected in Danon disease (LAMP2 cardiomyopathy) (Maron et al. 2009). As for other metabolic diseases, PAS-positive staining of lymphocytes, although not yet reported, might be present in oligosaccharidosis or nonlysosomal glycogen storage diseases. However, these disorders either differ in clinical symptoms, or PAS staining is not expected to be restricted to vacuoles.

Of note, the number of glycogen-filled lymphocyte vacuoles decreased rapidly after the start of treatment with recombinant human $\alpha$-glucosidase. Nevertheless, it is not likely to be a suitable marker for treatment efficacy, as there is no apparent link to clinical effects on heart, muscle, and liver (Winkel et al. 2003). In conclusion, we have shown that quantification of PAS-positive lymphocytes in peripheral blood films performs well in discerning patients with Pompe disease from controls. The test can be used as a reliable and simple screening method to support a diagnosis of Pompe disease.

Acknowledgements The authors thank all patients, parents, and blood donors for their contribution to the study, and I. de Coo, G. Ruijter, and F. Verheijen for their critical review of the manuscript. This research was supported by ZonMW- the Netherlands Organisation for Health Research and Development [project no. 152001005] and by the European Union, $7^{\text {th }}$ Framework Programme 'EUCLYD- A European Consortium for Lysosomal Storage Diseases' [health F2/ 2008 grant agreement 201678 to ATvdP and AJJR].

Open Access This article is distributed under the terms of the Creative Commons Attribution Noncommercial License which permits any noncommercial use, distribution, and reproduction in any medium, provided the original author(s) and source are credited.

\section{References}

Anderson G, Smith VV, Malone M, Sebire NJ (2005) Blood film examination for vacuolated lymphocytes in the diagnosis of metabolic disorders; retrospective experience of more than 2, 500 cases from a single centre. J Clin Pathol 58:1305-1310

Bain JB (1995) Blood cells a practical guide, 2nd edn. Blackwell Science, Oxford, pp 186-188

de Barsy T, Hers HG (1975) Biochemical and ultrastructural study of leucocytes in type II glycogenosis. Arch Int Physiol Biochim 83:954-955
Hayhoe FGJ, Quaglino D (1980) Carbohydrates. Haematological cytochemistry. Churchill Livingstone, New York, pp 50-67

Hirschhorn R, Reuser AJJ (2000) Glycogen Storage Disease type II; acid $\alpha$-Glucosidase (Acid Maltase) deficiency. In: Scriver CR, Beaudet AL, Sly W, Valle D (eds) 8th edn, vol III. Mc Graw-Hill, New York, pp 3389-3420

Ikeda K, Goebel HH, Burck U, Kohlschutter A (1982) Ultrastructural pathology of human lymphocytes in lysosomal disorders: a contribution to their morphological diagnosis. Eur J Pediatr 138:179-185

Maron BJ, Roberts WC, Arad M, Haas TS, Spirito P, Wright GB, Almquist AK, Baffa JM, Saul JP, Ho CY, Seidman J, Seidman CE (2009) Clinical outcome and phenotypic expression in LAMP2 cardiomyopathy. JAMA 301:1253-1259

Mitus WJ, Bergna LJ, Mednicoff IB, Dameshek W (1958) Cytochemical studies of glycogen content of lymphocytes in lymphocytic proliferations. Blood 13:748-756

Muntoni F, Zafeiriou DI, Jimenez C, Reuser A, Sewry C (2005) A case presenting with delayed motor milestones. Neuromuscul Disord 15:817-818

Okumiya T, Keulemans JL, Kroos MA, Van der Beek NM, Boer MA, Takeuchi H, Van Diggelen OP, Reuser AJ (2006) A new diagnostic assay for glycogen storage disease type II in mixed leukocytes. Mol Genet Metab 88:22-28

Straub V (2008a) 4. Diagnosis. In: Baethmann M, Straub V, Reuser AJJ (eds) Pompe disease. UNI-MEd Verlag AG, Bremen, pp 47-57

Straub V (2008b) 6. Differential diagnosis of Pompe disease. In: Baethmann M, Straub V, Reuser AJJ (eds) Pompe disease. UNIMEd Verlag AG, Bremen, pp 65-71

Trend PS, Wiles CM, Spencer GT, Morgan-Hughes JA, Lake BD, Patrick AD (1985) Acid maltase deficiency in adults. Diagnosis and management in five cases. Brain 108(Pt 4):845-860

van der Ploeg AT, Reuser AJ (2008) Pompe's disease. Lancet 372:1342-1353

van Diggelen OP, Oemardien LF, van der Beek NA, Kroos MA, Wind HK, Voznyi YV, Burke D, Jackson M, Winchester BG, Reuser AJ (2009) Enzyme analysis for Pompe disease in leukocytes; superior results with natural substrate compared with artificial substrates. J Inherit Metab Dis 32:416-423

von Bassewitz DB, Bremer HJ, Bourgeois M, Grobe H, Stoermer J (1977) Vacuolated lymphocytes in type II glycogenosis-a diagnostic approach? Eur J Pediatr 127:1-7

Winchester B, Bali D, Bodamer OA, Caillaud C, Christensen E, Cooper A, Cupler E, Deschauer M, Fumic K, Jackson M, Kishnani P, Lacerda L, Ledvinova J, Lugowska A, Lukacs Z, Maire I, Mandel H, Mengel E, Muller-Felber W, Piraud M, Reuser A, Rupar T, Sinigerska I, Szlago M, Verheijen F, van Diggelen OP, Wuyts B, Zakharova E, Keutzer J (2008) Methods for a prompt and reliable laboratory diagnosis of Pompe disease: report from an international consensus meeting. Mol Genet Metab 93:275-281

Winkel LP, Kamphoven JH, van den Hout HJ, Severijnen LA, van Doorn PA, Reuser AJ, van der Ploeg AT (2003) Morphological changes in muscle tissue of patients with infantile Pompe's disease receiving enzyme replacement therapy. Muscle Nerve 27:743-751

Winkel LP, Hagemans ML, van Doorn PA, Loonen MC, Hop WJ, Reuser AJ, van der Ploeg AT (2005) The natural course of nonclassic Pompe's disease; a review of 225 published cases. J Neurol 252:875-884 